\title{
The ion-driven permeation experiment PERMEX
}

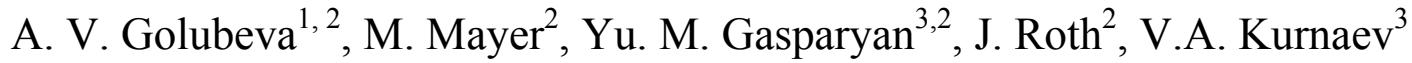 \\ ${ }^{1}$ - RRC 'Kurchatov Institute', Ac. Kurchatov sq., 1/1, Moscow RU-123182, Russia \\ ${ }^{2}$ - Max-Planck-Institut für Plasmaphysik, EURATOM Association, Boltzmannstr. 2, D-85748 \\ Garching, Germany \\ ${ }^{3}$ - Moscow Engineering and Physics' Institute, 115561, Kashirskoe shosse 31, Moscow, Russia
}

\begin{abstract}
A new installation PERMEX for the investigation of ion-driven permeation through metals was designed and built. A metal membrane under investigation separates two high-vacuum chambers. In the implantation chamber the membrane surface is irradiated with a monoenergetic deuterium ion beam with an incident energy in the interval of 100-3000 $\mathrm{eV} / \mathrm{D}$. The ion flux at the surface is in a range of $10^{13}-2 \cdot 10^{14} \mathrm{D} / \mathrm{cm}^{2} \mathrm{~s}$, the membrane temperature can be varied in the interval of $290-1050 \mathrm{~K}$. The permeating deuterium flux from the outlet membrane surface to the volume of the second chamber (registration chamber) is measured by a quadrupole mass-analyser. The outlet surface of the membrane can be cleaned by an argon ion beam. To approve the correct work of the setup a number of experiments were performed with Ni membranes. First results of experiments with tungsten membranes are presented.
\end{abstract}

\section{Introduction}

Hydrogen interaction with first-wall and constructive materials is one of the key aspects of thermonuclear reactor operation. Tritium permeation through the materials is a question of double interest: as a component of fuel recycling and due to radioactive safety of the reactor. While hydrogen gas-driven permeation is widely investigated for a number of 
materials, ion-driven permeation through materials is only sparsely investigated mainly due to the difficulties of ion-driven permeation experiments. Some data on hydrogen ion-driven permeation through polycrystalline tungsten are available [1, 2], but all existing data were obtained in the limited temperature interval $450 \div 850 \mathrm{~K}$ and the influence of the surface conditions on tungsten permeability was not investigated. The lack of experimental data on ion-driven permeation through materials was the reason for constructing the new experimental setup PERMEX for the investigation of ion-driven permeation through metals. The setup is intended to deal with materials with low permeability and particularly tungsten as one of the plasma-facing materials of ITER.

In laboratory experiments tritium-materials interaction is usually modelled by hydrogen or deuterium due to the difficulties of working with the radioactive tritium. The advantage of deuterium is its negligible background contribution under ultra-high vacuum conditions, in contrast to hydrogen. Consequently the experiments in the present work were performed with deuterium.

\section{Experimental setup}

A schematic representation of the PERMEX setup is shown in Fig. 1. An Oak Ridge type duopigatron ion source (1), described in [3], produces an initial ion beam in the energy range $1 \div 8 \mathrm{keV}$ per ion. After mass separation in a magnetic analyzer (2) the ion beam passes through an ion-optical system consisting of 4 pairs of deflection plates (3), an electrostatic lens (4) and a set of exchangeable diaphragms (5), and then irradiates the analysed membrane (7). The membrane separating implantation and registration vacuum chambers is mounted between the front and back parts of the sample holder and is vacuum sealed with gold gaskets (Fig. 2). The membrane has the shape of a disk with thickness of $0.05 \div 1 \mathrm{~mm}$, the sample area exposed in vacuum has a diameter of $27 \mathrm{~mm}$. In order to exclude hydrogen bypassing from the implantation to the registration chamber, for example through the gold sealings or through the stainless steel holder, the outer edge of the membrane is exposed to atmospheric pressure. There is no direct connection between the 
two chambers, except through the membrane. In order to suppress oxidation of the atmosphere-exposed part of the membrane at elevated temperatures, the atmospheric outer edge of the membrane is surrounded with a collar. The volume inside the collar is flushed with a constant flow of nitrogen gas, thus reducing the oxygen partial pressure (Fig. 2).

The sample holder is placed inside a horizontal split oven (Carbolite) (8), allowing to vary the membrane temperature in the interval $290-1273 \mathrm{~K}$. The oven temperature is measured by thermocouples in the oven and attached to the sample holder. It is stabilized with deviations below $2 \mathrm{~K}$ from the set point during tens of days. The uncertainty of the temperature measurement is $\sim 15 \mathrm{~K}$. An additional heating by the incident ion beam was not observed, as well as a drop in the permeation flux after "beam of" like in [4]. To achieve low incident ions energies the sample holder and oven can be biased with a decelerating voltage. For this purpose the sample holder is electrically insulated from other constructive elements via high-voltage ceramic breaks (6).

The hydrogen flux permeating through the membrane is measured by a quadrupole mass-analyser (QMS) (10) in the registration chamber. In case of a weak permeation signal the partial pressure of the permeating gas can be increased by reducing the pumping speed in the registration chamber by partially closing of a controllable valve (11) between the registration chamber and the pump. For absolute calibration of the QMS a set of calibrated leaks (9) with hydrogen and deuterium is used. An ion gun (12) allows to clean the back side of the membrane by $1.5 \mathrm{keV} \mathrm{Ar}{ }^{+}$ions.

\subsection{Vacuum system}

To provide the pressure gradient between ion source $(\sim$ mbar $)$ and implantation chamber $\left(\sim 10^{-6}\right.$ mbar $)$ a differential pumping system is used. The intermediate volume between the ion gun and the magnetic separator chamber is pumped with a Leybold Turbovac $1000 \mathrm{C}$ turbomolecular pump with a pumping speed of $850 \mathrm{l} / \mathrm{s}\left(\mathrm{N}_{2}\right)$.

In order to avoid back-diffusion of hydrogen from the implantation to the registration chamber through the vacuum system the pumping systems of inlet and outlet 
sides of the membrane were separated. The implantation chamber of the PERMEX setup has a usual pumping system consisting of a turbomolecular pump and a forepump. The registration chamber is pumped by a three-steps pumping system which consists of two concatenated turbomolecular pumps and a forepump. The consecutive connection of two turbomolecular pumps provides a higher compression level for gases.

The vacuum chambers can be heated up to $450^{\circ} \mathrm{C}$. After baking of the chambers at $150^{\circ} \mathrm{C}$ for 3 days and one day keeping the sample holder at a temperature $\sim 600{ }^{\circ} \mathrm{C}$ the residual gas pressure at the working temperature is better than $5 \times 10^{-9}$ mbar in the implantation chamber and $2 \times 10^{-9} \mathrm{mbar}$ in the registration chamber. During IDP experiments the deuterium gas pressure in the implantation chamber is $\sim 10^{-6}$ mbar as a result of gas flow from the ion source. For the measurement of gas driven permeation the gas pressure in the implantation chamber can be increased.

\subsection{Ion-optical system}

A duopigatron ion source provides an intensive ion beam in the energy range of $1 \div 8 \mathrm{keV}$. In order to achieve lower incident energies a deceleration of the beam is required. The deceleration system consists of four pairs of deflection plates and an electrostatic lens. Due to space restrictions (the sample holder is heated under high voltage in the furnace) it was not possible to place the focusing system directly in front of the membrane. The lens is placed at a distance of $300 \mathrm{~mm}$ from the membrane. The deflection plates are placed in front of the lens. The later is justified by the good alignment of the whole setup which usually doesn't require a strong deflection for focusing on the sample. As focusing lens the classical three-electrodes cylindrical lens with inner diameter of $20 \mathrm{~mm}$ and $2 \mathrm{~mm}$ distance between electrodes was selected.

The ion beam optics of the focusing system was modelled with the SIMION 7 code, which calculates charged particles trajectories in electrostatic fields of electrodes, taking space charge effects into account. Based on this modelling a suitable electrodes geometry and arrangement was chosen, and optimal electrode potentials were selected for different 
incoming ion energies in order to provide best ion beam focusing on the membrane. Fig. 3 illustrates ions trajectories in case of slowing down of $3.6 \mathrm{keV} \mathrm{D}_{3}{ }^{+}$ion beam by $3 \mathrm{kV}$ sample potential to irradiate the membrane with $600 \mathrm{eV} \mathrm{D}_{3}{ }^{+}$(200 eV deuteron). A set of exchangeable diaphragms is used in order to cut-off the ion beam before entering the sample holder (point " $x$ " on Fig. 3) in order to have an additional control of the beam diameter. These diaphragms consist of double apertures: the front aperture is at ground potential, while the back one is biased to $-100 \mathrm{~V}$ in order to suppress secondary electrons. The experiments showed a good focusing of the ion beam with usage of the potentials chosen by modelling.

To determine the ion beam spot size sputtering of a $300 \mathrm{~nm}$ thick hard a-C:H film deposited on stainless steel was used. The colour of these films strongly depends on their thickness, and changes due to sputtering under ion bombardment. The ion beam spot size had a diameter of $6 \mathrm{~mm}$ at the initial energy of $1 \mathrm{keV} / \mathrm{D}$ and $15 \mathrm{~mm}$ at $\mathrm{E}_{0}=200 \mathrm{eV} / \mathrm{D}$. In case of $200 \mathrm{eV}$ deuterons the beam size spot is larger because of repulsing of positive ions in the ion beam, playing a more pronounced role in case of low energies (beam deceleration by membrane potential). In the energy interval of $200 \mathrm{eV}-3 \mathrm{keV}$ the irradiated area is $2-4$ smaller than the inner diameter of the sample holder $(24 \mathrm{~mm})$. The uncertainty of the ion beam current measurement is about $10 \%$.

The spatial current distribution in the beam spot was determined at the tandem accelerator at IPP, Garching, Germany by nuclear reaction analysis (NRA) using the reaction

$$
\mathrm{D}+\mathrm{He}_{3} \rightarrow \mathrm{He}_{4}+\mathrm{p}
$$

The protons generated in the reaction were registered. The beam of $800 \mathrm{keV} \mathrm{He}{ }_{3}^{+}$ions had $\varnothing 1 \mathrm{~mm}$, giving the spatial resolution of the analysis. The obtained ion beam profile for a tungsten sample implanted in the PERMEX setup with $1.2 \mathrm{keV}$ deuterons is shown in Fig. 4. It is close to a Gaussian distribution, and the half-width of the distribution matches the visual estimation of the ion beam spot size by the a-C:H film erosion. In the interval $200 \mathrm{eV} \div 3 \mathrm{keV} / \mathrm{D}$ of ion energies the ion current on the sample was about $2 \cdot 10^{-6} \mathrm{~A}$, 
corresponding to an incident deuterium flux on the surface of $\sim 10^{13} \mathrm{D} / \mathrm{cm}^{2}$ s with decelerated ion beam and $\sim 1 \cdot 10^{14} \mathrm{D} / \mathrm{cm}^{2} \mathrm{~s}$ without deceleration.

\subsection{Ion current measurement}

As described above, experiments with low ion energies require applying a deceleration potential to the sample. Sample holder, surrounding oven and current integrator are insulated from ground, the electrical supply of the oven and smaller devices (such as current integrator) goes through an isolated transformer. The sample holder is a tube with a membrane at the bottom. The relation of sample holder length to sample diameter is $\sim 10: 1$, so the whole construction acts as a Faraday cap collecting most of the secondary electrons and providing an accurate current measurement with an estimated inaccuracy below $10 \%$. The ion beam current is measured with a current integrator of type ORTEC 439.

\subsection{Cleaning the back side of the membrane}

An ion gun type 04.303A manufactured by Perkin Elmer is mounted in the analysing chamber. It allows to produce an argon ion beam with an energy in the range $1 \div 5$ $\mathrm{keV}$ bombarding the membrane outlet surface. Typically the Ar ions are extracted from the ion gun at $2.5 \mathrm{keV}$ with a sample deceleration potential of $1 \mathrm{keV}$, giving an incident argon ions energy of $1.5 \mathrm{keV}$. These conditions allow us to focus the ion beam on the membrane under investigation without touching the sample holder. The argon flux at the surface is $10^{13} \mathrm{Ar}^{+} / \mathrm{cm}^{2} \mathrm{sec}$, and the ion beam spot situated in the middle of the sample has a round shape with an area of $\sim 3 \mathrm{~cm}^{2}$. During argon irradiation the pressure in the analysing chamber increases to $\sim 5 \cdot 10^{-7}$ mbar because of a neutral gas coming from the ion gun. In our experiments the back side of the membrane was initially cleaned for one hour after membrane installation, and then an additional optional 10 minutes cleaning could be performed between implantations. This procedure allowed us to get permeation curves reproducible within $10 \%$ of amplitude in $\mathrm{W}$ experiments. An oxidation afterward is possible, but accordingly to our estimations at the working conditions of present 
experiments a typical oxidation time is more than 50 hours, so argon beam irradiation has a long-time effect on the surface conditions at the backside of membrane.

\subsection{QMS calibration}

The signal of deuterium permeating in the analyzing chamber was measured with a quadrupole mass-analyzer type QMS 422 by Pfeiffer Vacuum, working in ion count mode. It has a high sensitivity for hydrogen of $10^{-14}$ mbar, allowing to perform ion-driven permeation experiments even with materials having very low permeability, such as tungsten.

At high-vacuum conditions deuterium which penetrates through the membrane is released from the surface as $\mathrm{D}_{2}$ or $\mathrm{HD}$ molecules.

To measure the absolute permeation flux the QMS is calibrated with a set of calibrated capillary $\mathrm{D}_{2}$ leaks with fluxes of $1.8 \cdot 10^{-13} \mathrm{~mol} / \mathrm{s}, 1.9 \cdot 10^{-12} \mathrm{~mol} / \mathrm{s}$ and $8.0 \cdot 10^{-11}$ mol/s (10\% uncertainty) produced by Vacuum Technology Incorporated and with the calibrated $\mathrm{H}_{2}$ leak with the flux of $8.6 \cdot 10^{-11} \mathrm{~mol} / \mathrm{s}$ (3.8\% uncertainty) manufactured by LACO Technologies.

The calibrated leaks are situated close to the membrane, resulting in equal pumping speeds for deuterium fluxes coming through the membrane and from the calibrated leaks.

As QMS sensitivity for $\mathrm{HD}$ an average between $\mathrm{H}_{2}$ and $\mathrm{D}_{2}$ sensitivities was used. The calibrations showed a linear dependence of the QMS signal from the flux in the range $1 \cdot 10^{-11} \div 2 \cdot 10^{-13} \mathrm{~mol} / \mathrm{s}$. Calibration is usually performed after every new membrane installation.

\section{Experiments}

After membrane installation and pump down a QMS calibration is performed. Then typically several days are spent for baking the chamber in order to remove deuterium introduced during calibration and improve vacuum conditions by reducing the partial pressure of water. Finally, before sample heating, the residual pressure in both chambers better than $5 \cdot 10^{-9}$ mbar is achieved. With increasing membrane temperature the working pressure in the chamber slightly increases up to $2 \cdot 10^{-8}$ mbar at $700^{\circ} \mathrm{C}$. A several days 
annealing leads to a restore of the initial good vacuum conditions. After achieving steady residual pressure in the registration chamber experiments can be performed.

To increase the deuterium flux on the inlet surface membrane is irradiated with $\mathrm{D}_{2}{ }^{+}$ or $\mathrm{D}_{3}{ }^{+}$ions. During permeation experiment the QMS in the registration chamber registers the signal of $\mathrm{HD}, \mathrm{D}_{2}, \mathrm{HDO}, \mathrm{D}_{2} \mathrm{O}$ and several constituents of the residual gas.

\section{Experimental results}

\subsection{Nickel}

The deuterium permeation through nickel has been investigated more extensively than other metals in the literature due to the high hydrogen permeability of nickel. Due to this fact measurements of the nickel permeability are a good test for the performance of the PERMEX setup. The other advantage of nickel is, that at the working conditions of Permex (working pressure at inlet membrane side $\sim 10^{-6} \mathrm{mbar}$ ) the gas-driven permeating flux can be fixed, so that both gas- and ion-driven deuterium permeations (GDP and IDP) through nickel can be studied.

A nickel foil of $99.5 \%$ purity was used. The samples were first mechanically and then electrolytically polished and had a thickness of about $0.375 \mathrm{~mm}$. The deuterium permeation rate was measured at an incident energy of $1 \mathrm{keV} / \mathrm{D}$ and in the temperature range $523-773 \mathrm{~K}$. In the experiments the level of gas-driven permeating flux was fixed before and after membrane irradiation with deuterium ions.

The gas-driven permeation regime in the given temperature range is supposed to be diffusion-limited [5]. This was approved in the present work by the fact that argon beam cleaning of the membrane back side did not change the permeating flux. So for the ion-

driven permeation regime two variants are possible: it can be either limited by recombination at a front side and diffusion at a back side (RD) or limited by diffusion at both surfaces (DD). The relations of permeating and incident fluxes for these cases are [6]:

$$
\Phi=\frac{r \cdot D_{b}}{L \cdot D_{f}} \alpha \cdot \Phi_{i},
$$




$$
\Phi=\frac{D_{b}}{L \sqrt{k_{f}}} \sqrt{\alpha \cdot \Phi_{i}},
$$

where $\Phi$ - permeating flux, $\Phi_{i}$ - implanting flux, $\alpha$ - implantation coefficient, $D$ - diffusion coefficient, $k$ - recombination coefficient, $r$ - mean implantation depth, $L$ - membrane thickness. Indexes $f, b$ correspond to front and back surfaces of the membrane.

At $1 \mathrm{keV}$ incident energy and $\mathrm{T}<550 \mathrm{~K}$ the permeation in the ion-driven regime was found to be limited by recombination at the inlet surface and by diffusion at the outlet surface [7]. In our experiments the ratio of incident and permeating fluxes is $\Phi / \Phi_{i} \sim 0.01$. At this ratio of fluxes supposing DD-regime from (1) one obtains $\frac{D_{b}}{D_{f}} \sim 100$. Ion beam irradiation can either increase or does not change the diffusion coefficient in the implantation region. Therefore, $\mathrm{RD}$ permeating regime should be the case of our experiments.

A typical permeation curve of deuterium GDP through nickel is presented in Fig. 5. After loading the inlet side with deuterium gas the permeating flux appears after a few seconds of delay, grows, and then reaches steady-state. No spike on the permeating curve is present in the case of GDP.

In contrast to GDP, the permeating curve of deuterium IDP through nickel often has a spike at the beginning of the irradiation. Several IDP permeating curves obtained at the same temperature in a set of consistent measurements are presented on Fig. 6. The permeation spike is present at the first few implantations, becomes smaller with each implantation until it disappears. An interruption of the irradiation leads to a partial restoration of the spike. After the initial spike the permeating flux reaches a quasi steadystate level, slightly dropping with time (about $10 \%$ overnight). The absence of a true steadystate permeation level is typical for nickel irradiated with energetic hydrogen [8].

Such a behavior (an initial spike and slight drop after) is typical for hydrogen IDP through Ni [9]. The nature of the spike is not completely clarified until now. Impurities on the inlet surface $(\mathrm{O}, \mathrm{C})$ may suppress the reemission flux, thus increasing the permeation 
flux. After the impurities on the inlet side are sputtered by the ion beam, the permeating flux drops. The recovery of the spike is explained by deposition of impurities from the residual gas on the membrane surface. Another suggestion is, that the ion beam creates defects catalyzing recombination on the front surface or creating channels for quick diffusion of implanted atoms to the front surface of the membrane. The restoration of the spike is explained by annealing of defects with time [10]. Both reasons may act together [11]. Some authors explain the spike by diffusion of impurities (mainly $\mathrm{S}, \mathrm{O}, \mathrm{Si}$ ) from the bulk of nickel at elevated temperatures [12], which are then removed by the incident ion beam.

Fig. 6 shows a good repeatability of the quasi-steady-state part of the IDP curve in similar experiments. It should be mentioned, though, that during experiments the surface conditions are constantly changing due to sputtering of impurities creation of defects in the implantation zone, resulting in easier reemission of implanted deuterium. Consequently, on a time scale of weeks a pronounced decrease of the ion-driven permeating flux at identical conditions is observed.

As for the gas-driven permeation, the permeating flux value was repeatable within a 2 weeks experimental campaign. The reason is that the membrane area exposed to gas is much larger (about 24 times) than the ion beam spot, so ion-beam modification of a small fraction of the surface does not influence GDP pronouncedly.

The GDP and IDP fluxes grow with temperature. The permeating fluxes, as obtained after about one hour after reaching the quasi-steady state permeation, are shown in Fig. 7. The large scatter of IDP data can be explained by a constant change of the inlet surface conditions due to the ion beam irradiation and the quasi-stationary character of permeation curve. The activation energies of IDP and GDP were estimated as 0.3 and $0.5 \mathrm{eV}$. These values are in the range of existing data on the nickel permeability: $0.13-0.48$ $\mathrm{eV}$ for deuterium ion- and plasma-driven permeation [8,7, 13], 0.49-0.58 eV for hydrogen gas-driven permeation $[5,14]$. 


\subsection{Tungsten}

A $50 \mu \mathrm{m}$ tungsten foil of $99.97 \mathrm{wt} . \%$ purity produced by Plansee AG was used. The main impurities are Mo $(<100 \mu \mathrm{g} / \mathrm{g}), \mathrm{Fe}, \mathrm{C}(<30 \mu \mathrm{g} / \mathrm{g}), \mathrm{Cr}$, Ta, O, Si, P $(<20 \mu \mathrm{g} / \mathrm{g}), \mathrm{Ag}, \mathrm{Co}, \mathrm{K}$, $\mathrm{Na}, \mathrm{Cu}, \mathrm{Nb}(<10 \mu \mathrm{g} / \mathrm{g})$. After membrane cutting and ultrasonic cleaning no additional treatment was performed, except an optional cleaning of the outlet membranes surfaces by argon bombardment. NRA analyses showed the presence of oxygen and carbon at the surface. At normal conditions tungsten is always covered with a $\mathrm{WO}_{2}$ layer, which is proved by XPS analysis. So the measured oxygen impurity of $\sim 2.5 \cdot 10^{16} \mathrm{O} / \mathrm{cm}^{2}$ corresponds to about $4 \mathrm{~nm}$ thick $\mathrm{WO}_{2}$. There may be also some water present. The carbon very probably originates from hydrocarbons (for example oil), but may be also adsorption of $\mathrm{CO}$ or $\mathrm{CO}_{2}$. The permeability of the membranes was investigated at irradiation with $\mathrm{D}_{3}{ }^{+}$ions at an energy of $200 \mathrm{eV} / \mathrm{D}$ (which is below the threshold energy for creating Frenkel pairs in tungsten) in the temperature range of $823 \div 973 \mathrm{~K}$. The irradiating ion flux was equal to $F_{0}=$ $1.5 \cdot 10^{13} \mathrm{D} / \mathrm{cm}^{2} \mathrm{sec}$. One should take into account, that only a fraction of the incident flux $\left(0.36 F_{0}\right.$ according to SCATTER calculations [15]) penetrates into the material, while other particles are backscattered from the surface of the membrane. For a more visual representation of the results we introduce an effective permeability coefficient $F / F_{0}$, were $F$ is the permeating flux.

Eight membranes were investigated. The largest number of experiments with different membranes at the same conditions is 4 , the largest number of experiments with the same sample at different temperatures is 9 . The scatter of the permeating flux for the selected sample goes into $\pm 10 \%$ interval from the mean value $\bar{F}$, while the scatter for the different membranes is three times larger, which can be probably explained by a scatter of sample properties.

Permeating deuterium desorbs as $\mathrm{HD}$ and $\mathrm{D}_{2}$ molecules. Before proper vacuum chambers annealing it is also possible to observe deuterium contained in water molecules as HDO, but after chamber annealing the water signals disappear. 
A typical permeation curve is shown at the Fig. 8. The irradiation with $200 \mathrm{eV} / \mathrm{D}$ was started at $\mathrm{t}=0$. During a delay time $\tau$, necessary for diffusion of thermalized particles though the foil, no permeating signal can be seen. The delay time is defined as the intersection of the steepest slope tangent of the permeation curve with the x-axes. After appearance, the signal rises and reaches steady-state. The constant signal through ten hours of experiment suggests constant surface conditions. After stopping the implantation the permeating signal does not drop immediately but with a delay close to $\tau$. These features of the permeating curve are typical for hydrogen ions implantation into tungsten [16].

In the present work the influence of the conditions at the back side of the tungsten membrane on tungsten permeability was investigated for the first time. The results are illustrated by Fig. 9, where the effective permeability coefficient after reaching steady-state is plotted via the inverse temperature. The outlet surface of the membrane $a$ was not cleaned by the argon beam. With increasing temperature the permeating flux increases and is about $\sim 5 \cdot 10^{-4} \mathrm{~F}_{0}$ in the temperature range of 873-933 $\mathrm{K}$.

Before the fist experiment the outlet surface of membrane $b$ was also not cleaned by argon irradiation, and the permanent flux at $\mathrm{T}=873 \mathrm{~K}$ through membrane $b$ is close to that of membrane $a$ at the same conditions. Then the outlet surface of the membrane was cleaned by irradiation with $\mathrm{Ar}^{+}$ions of $1.5 \mathrm{keV}$. The next permeation experiment at the same conditions showed a permanent permeating flux about 5 times higher than before cleaning. Further experiments $(b 2)$ demonstrated that for membrane $b$ the value of permeating flux remains about 5 times higher than for membrane $a$. At the end the temperature was decreased to initial $823 \mathrm{~K}(\mathrm{~b} 3)$ and the value of permeating flux was the same as just after argon cleaning, which let us suppose that during the described set of experiments with membrane $b$ (three days) the conditions responsible for the membrane permeability are unchanged.

The effect of membrane permeability increase after cleaning of the back side (as well as permeability drop after cleaning of the front side) was observed previously (but never with tungsten), and several explanations are proposed by different investigators: 
1) Damage by ion irradiation may cause rapid diffusion due to formation of short diffusion paths in implantation region [17].

2) The surface defects created by ion irradiation may catalyze hydrogen recombination and desorption of molecules from the surface, and thus increase permeating flux [11].

3) The impurities at the back surface may serve as an effective barrier for the permeation of implanted deuterium. The removal (total or partial) of impurities leads to an increase of the hydrogen flux through the cleaned surface $[11,18]$.

Under the conditions of our experiments the layer modified by argon bombardment is far $\left(5 \cdot 10^{3}\right.$ times) thinner than the membrane, so that even a faster hydrogen diffusion through the modified layer cannot strongly change the permeating flux.

The next explanation is the possibility of faster recombination and desorption on defects created during cleaning by the argon beam. Our experiments were carried out at $\sim 0.25$ of the melting temperature. At these temperatures interstitial atoms and vacancies become mobile and both defects annihilation and agglomeration of vacancies in clusters are possible. The observation of a constant permeating flux over days means that either defects created by argon cleaning agglomerate to stable clusters or they annihilate within a short time after the cleaning process.

The removal of impurities is also a possible reason for the increase of the permeating flux. As mentioned above, we initially find an oxide layer and carbon impurities at the surface under normal conditions. According to SRIM calculations, argon irradiation during 1 hour sputters $\mathrm{C}$ and $\mathrm{O}$ from sub-surface layer, thus increasing the permeating flux. Similarly, introducing impurities at the inlet membrane surface increases the permeating flux because it suppresses recombination and gas release from the inlet surface. It was shown in [19] that the presence of oxygen at the front surface of a nickel membrane increases the permeating flux by a factor of 2.5 , and carbon impurities by factor of about 4 . Introducing the same impurities at the outlet surface should analogously decrease the permeating flux. 
The pronounced increase of the permeating flux after outlet surface cleaning demonstrates the strong influence of surface conditions on tungsten permeability. This fact is important, especially for safety reasons, in the context of using tungsten as a plasma-facing material for fusion reactors, because the permeation of tritium through tungsten may depend strongly on the conditions at the tungsten back side.

\section{Conclusions}

A new setup PERMEX for the experimental investigation of ion-driven permeation through metal membranes was developed and constructed. It allows to measure permeation through metal membranes with thicknesses in the range $0.05 \div 1 \mathrm{~mm}$ and low permeability. A wide temperature interval of operation $(\mathrm{RT} \div 1050 \mathrm{~K})$ is available. The set-up allows cleaning of the membrane back side by sputtering with energetic argon ions. Test experiments with nickel showed results in reasonable agreement with existing literature, approving the correct operation of the set-up.

The ion-driven permeation of deuterium through $50 \mu \mathrm{m}$ thick polycrystalline tungsten foils was investigated in the temperature range from 823 to $1023 \mathrm{~K}$ at an implantation energy of $200 \mathrm{eV} / \mathrm{D}$. The influence of cleaning of the tungsten membrane back side on the permeation rate was investigated. Cleaning of the back side by $1.5 \mathrm{keV} \mathrm{Ar}^{+}$ results in an increase of the permeating flux by a factor of about 5 . This effect can be explained by removal of the oxide layer and carbon impurities from the back side, introduction of defects by argon sputtering, or a combination of both reasons.

\section{Acknowledgements}

The authors are very grateful to Dr. Denis Levchuk for his helpful consultations concerning designing and operation of PERMEX. We are also thankful to Dipl.-Ing. B. Plöckl for the engineering, R. Lang for the construction of the setup, P. Matern for the provision of electrical connections and automation of the pumping system, and A. Weghorn and M. Fusseder for the technical assistance. 
This work was partly supported by the Impuls- und Vernetzungsfond der Helmholtz Gemeinschaft, grant HRJRG-022. 


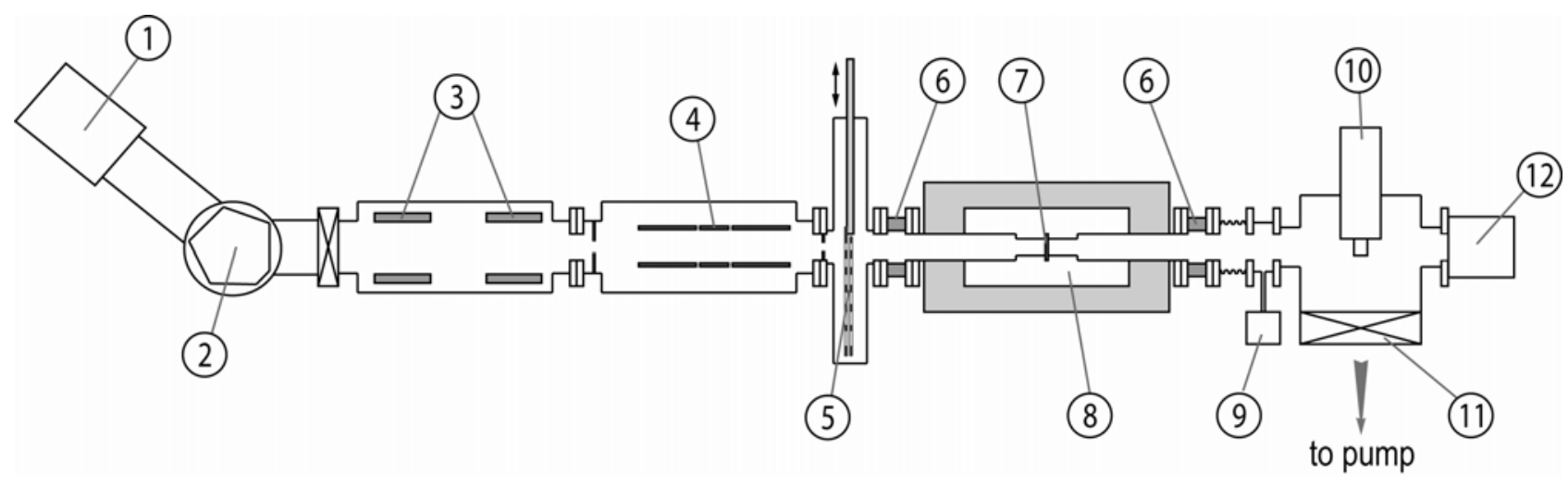

Fig. 1. Schematic representation of the PERMEX setup: 1 - ion source, 2 - magnetic massseparator, 3 - deflection plates, 4 - electrostatic lens, 5 - set of exchangeable diaphragms, 6 - high voltage insulators, 7 - membrane, 8 - heated area, 9 - calibration leaks, 10 - quadrupole massanalyzer, 11 - controllable valve, 12 - argon ion gun. 


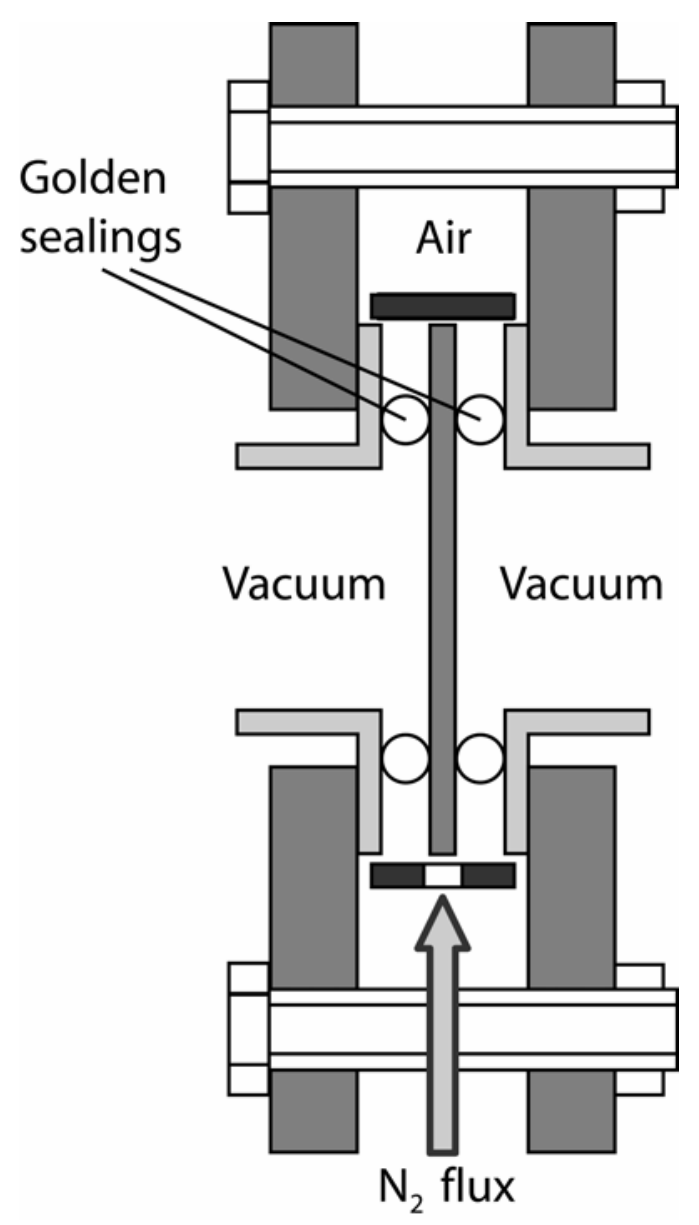

Fig. 2. Schematic representation of mounting and vacuum sealing of the membrane 


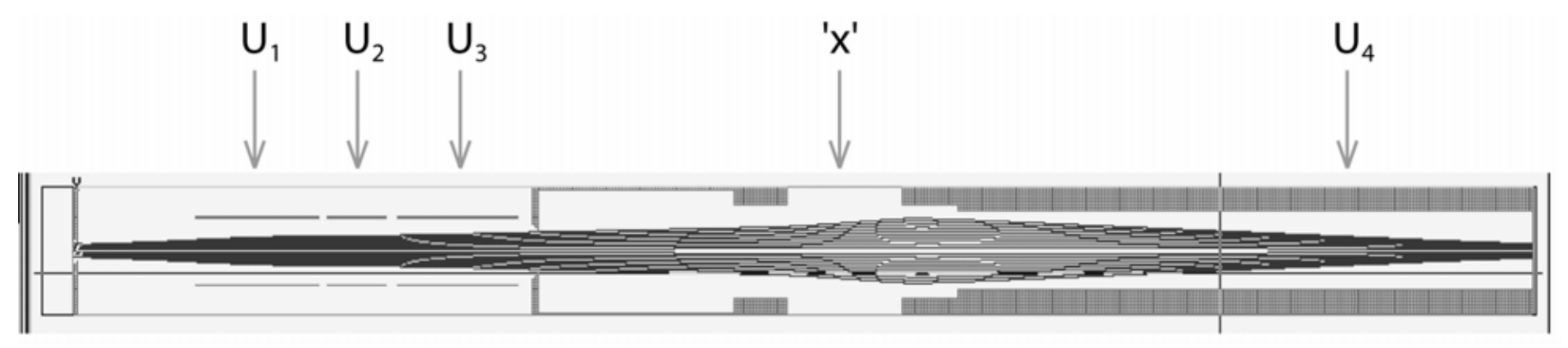

Fig. 3. $\mathrm{D}_{3}{ }^{+}$trajectories, as calculated with SIMION7, in the ion-optical system of the PERMEX setup. $\mathrm{E}_{0}=3.6 \mathrm{keV}, \mathrm{M}=6\left(\mathrm{D}_{3}{ }^{+}\right)$, initial angle divergence of beam entering the system $4^{0}$. Optimal potentials selected $-\mathrm{U}_{1}=+450, \mathrm{U}_{2}=+1600, \mathrm{U}_{3}=0, \mathrm{U}_{4}=+3000$. " $\mathrm{x}$ " - point where additional diaphragms are placed 


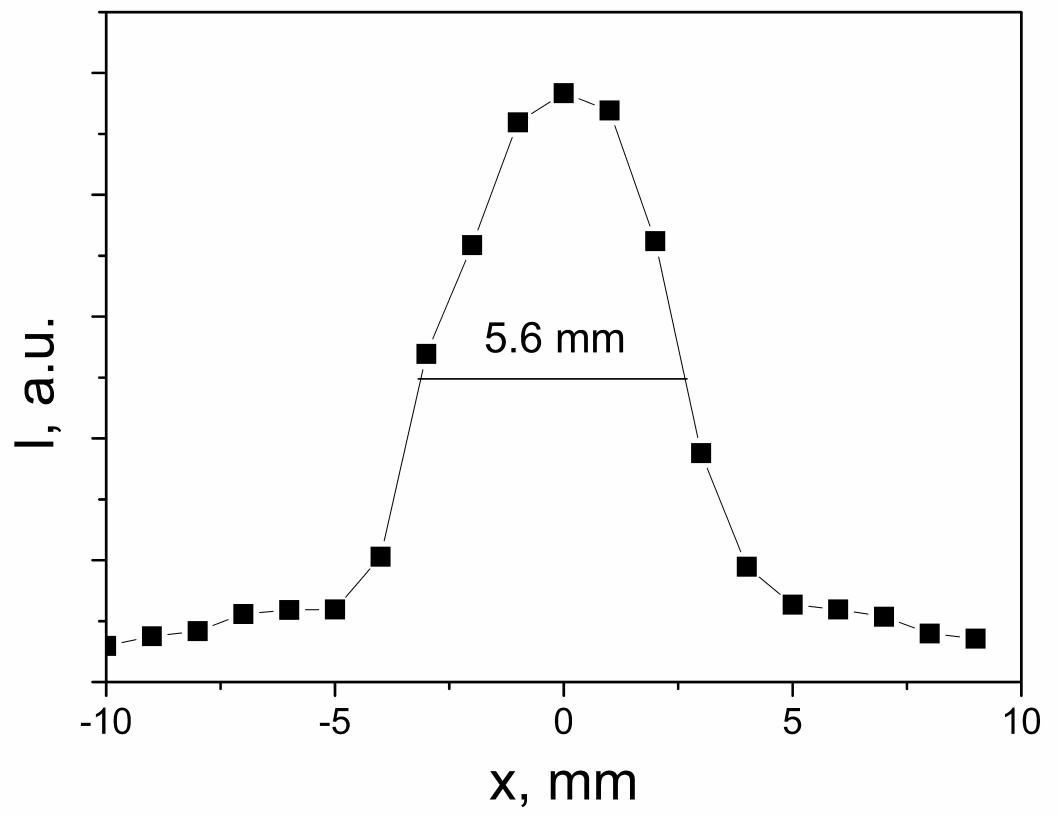

Fig. 4. Beam profile of $1.2 \mathrm{keV}$ deuterons, as obtained by the NRA method 


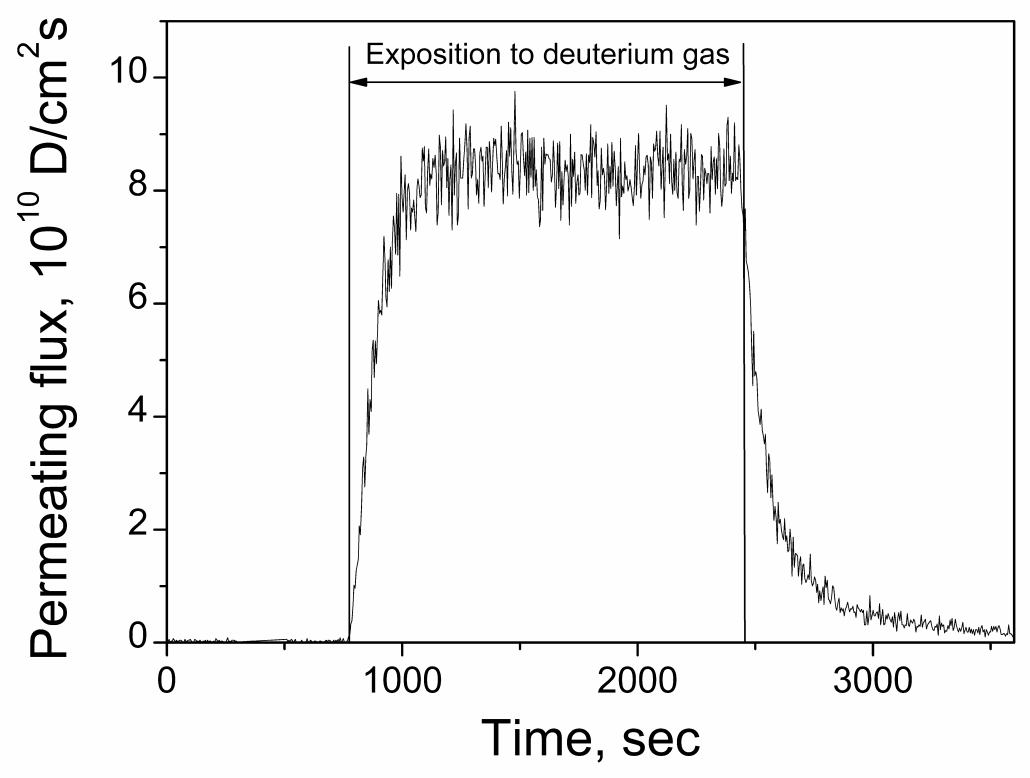

Fig. 5. Hydrogen gas-driven permeation through nickel: Permeation curve as obtained by exposing the inlet surface to deuterium gas, $\mathrm{P}=8 \cdot 10^{-6} \mathrm{mbar}, \mathrm{T}=773 \mathrm{~K}$. 


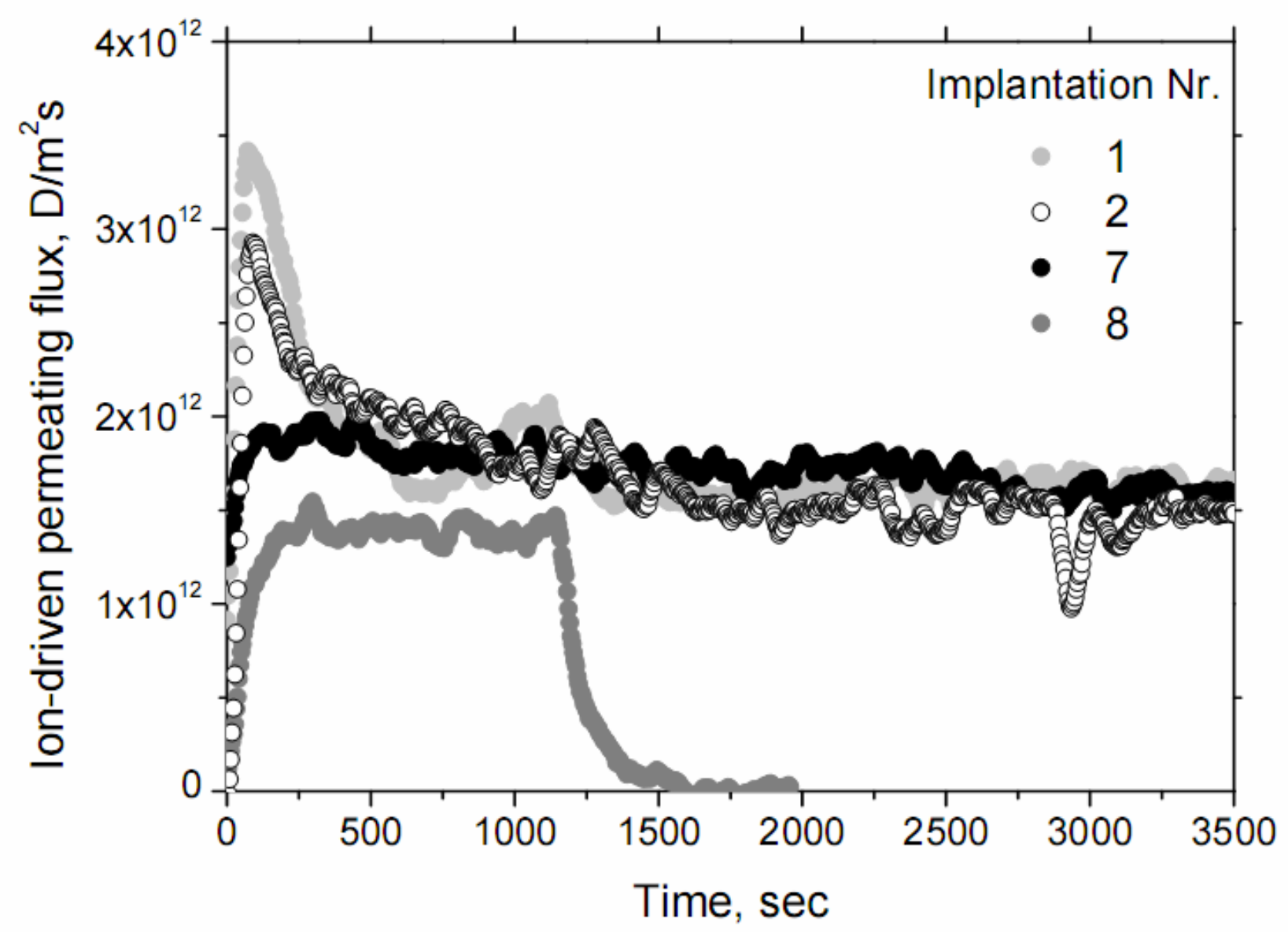

Fig. 6. A sequence of ion permeation curves (with gas-driven permeation subtracted background from the total permeating flux) in Ni. $\mathrm{E}_{0}=1 \mathrm{keV} / \mathrm{D}, \mathrm{T}=773 \mathrm{~K}$, incident flux $\mathrm{F}=1.3 \cdot 10^{14}$ $\mathrm{D} / \mathrm{cm}^{2} \mathrm{~s}$ The total number of implantations was 8 , from which only the first two and the last two are shown 


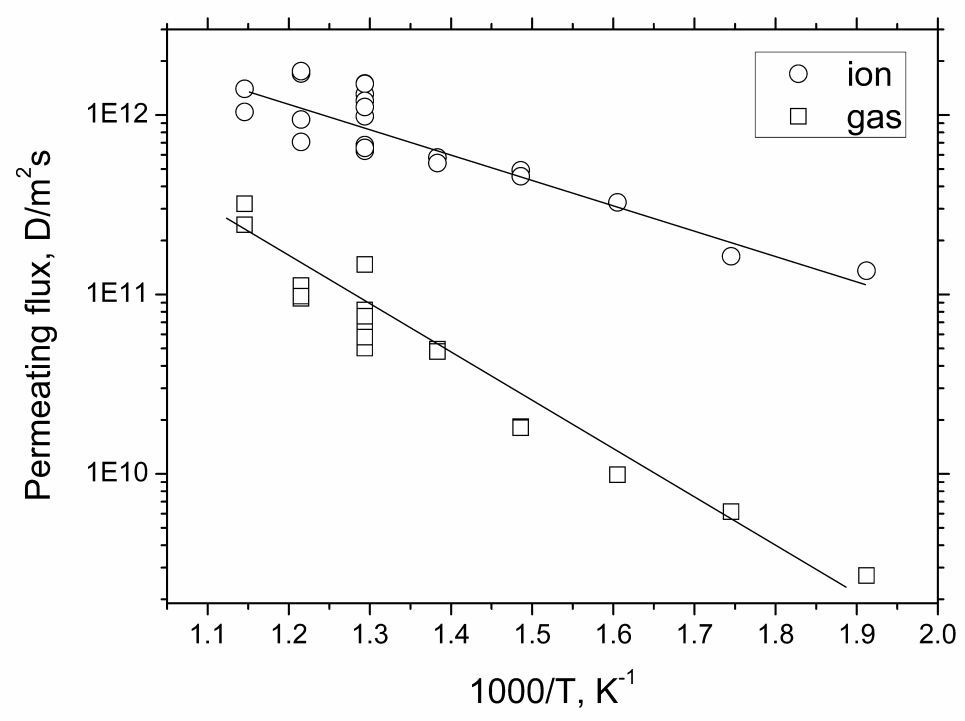

Fig. 7. Steady-state ion-driven (opened symbols) and gas-driven (closed symbols) permeation fluxes on reversed temperature at irradiation of a nickel membrane with $1 \mathrm{keV}$ deuterons in three sets of measurements. $\mathrm{J}_{0}=1.3 \cdot 10^{14} \mathrm{D} / \mathrm{cm}^{2} \mathrm{~s}$, gas loading of irradiated side $\sim 8 \cdot 10^{-6}$ mbar 

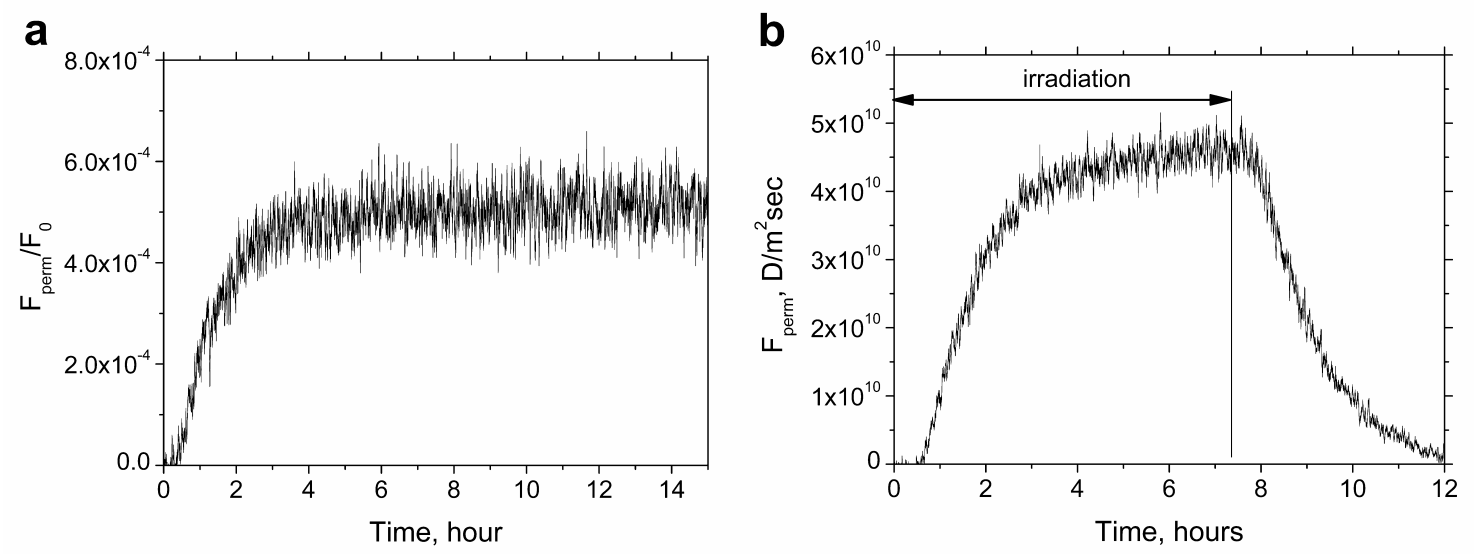

Fig. 8. Time dependence of deuterium flux permeating though the $50 \mu \mathrm{m}$ tungsten foil with a backside cleaned by an argon beam. $\mathrm{E}_{0}=200 \mathrm{eV} / \mathrm{D}, \mathrm{F}_{0}=1.5 \cdot 10^{13} \mathrm{D} / \mathrm{cm}^{2}$. a $-\mathrm{T}=855 \mathrm{~K}, \mathrm{~b}-\mathrm{T}=833$ K. 


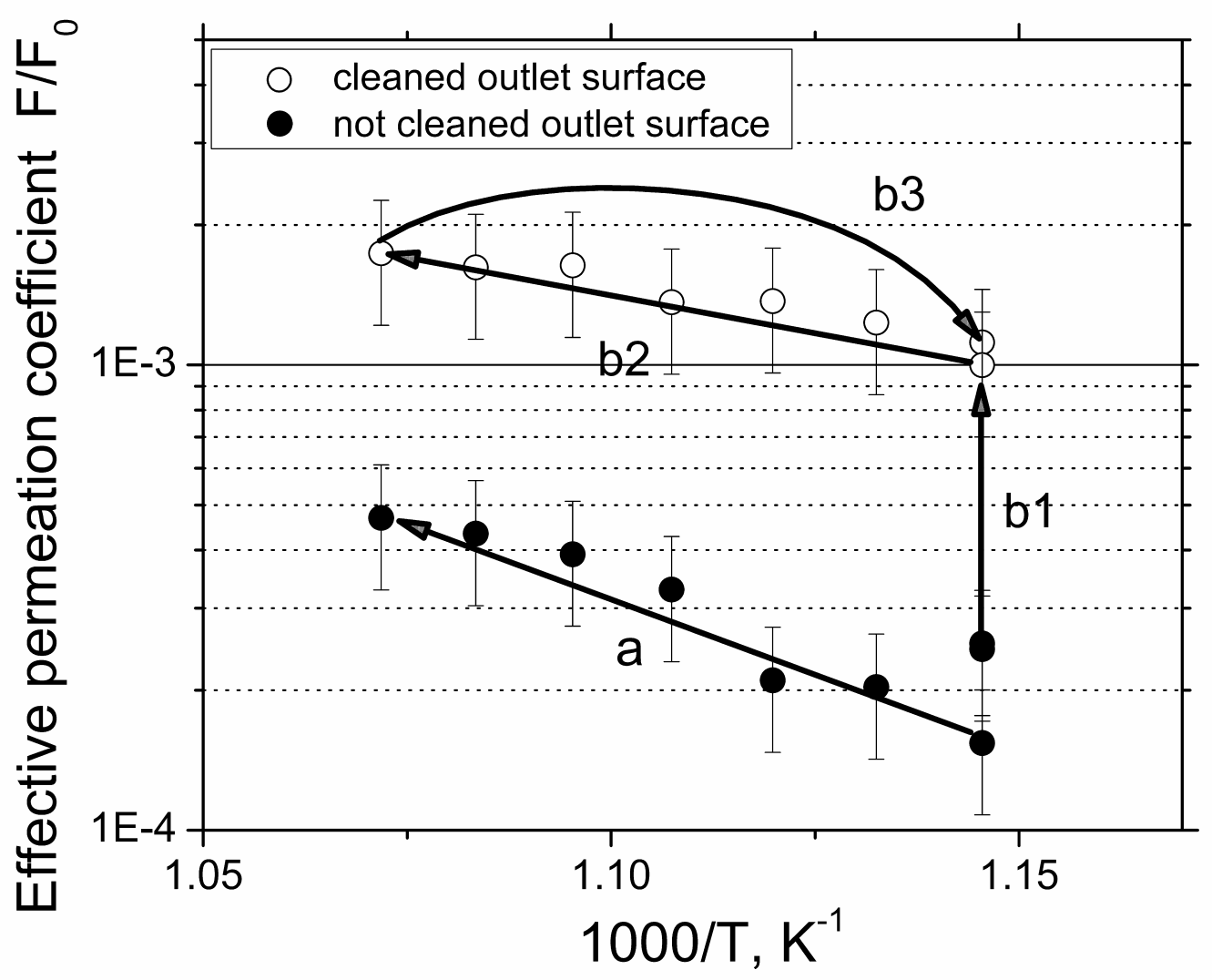

Fig. 9. The influence of membrane outlet surface cleaning on deuterium ion-driven permeation: the temperature dependence of the steady-state value of the permeating flux at membrane irradiation with $\mathrm{D}_{3}{ }^{+} . E_{0}=200 \mathrm{eV} / \mathrm{D} . F_{0}=1.5 \cdot 10^{13} \mathrm{D} / \mathrm{cm}^{2}$. Sample $a$ - not cleaned, sample $b$ - the outlet surface was cleaned with an argon beam after first irradiation with $\mathrm{D}_{3}^{+}$ 


\section{References}

1. R.A. Anderl, D.F. Holland, G.R. Longhurst, R.J. Pawelko, C.L. Trybus, C.H. Sellers // Fusion Technol. 21 (1992) 745-752

2. H. Nakamura, T. Hayashi, Y. Iwai, M. Nishi // Fusion Technol. 39 (2001) 894-898

3. Eckstein, W., Garcia-Rosales, C., Roth, J., and Ottenberger, W., "Sputtering data", IPP-report 9/82, 1993.

4. R.A. Anderl, D.F. Holland, G.R. Longhurst, J. Nucl. Mater. 176-177 (1990) 683-689

5. T. Namba, S. Kokubo, M. Kanno // J. of the Japan Institute of Metals, 42[4] (1978)

6. D.K. Brice, B.L. Doyle, J. Vac. Sci. Technol. A5 (1987) 2311-2314.

7. H. Nakamura, W. Shu, T. Hayashi, M. Nishi // J. Nucl. Mater. 313-316 (2003) 679-684

8. T. Tanabe, Y. Furuyama, H. Hirano and S. Imoto // J. Nucl. Mater., 128-129 (1984) 641-645

9. T. Tanabe, Y. Furuyama, S. Imoto // J. Nucl. Mater. 123 (1984) 1563-1567

10. T. Nagasaki, R. Yamada, H. Ohno // J. Vac. Sci. Echnol. A10 (1) (1992) 170.

11. V. Bandurko, K. Ohkoshi, K. Yamaguchi, M. Yamawaki, N. Koborov, V. Kurnaev, D. Levchuk // Vacuum 47, 6-8 (1996) 947-950.

12. M. Yamawaki, K. Yamaguchi, S. Tanaka, T. Namba, T. Kiyoshi and Y. Takahashi // J. Nucl. Mater., 162-164 (1989) 1071-1076.

13. V. M. Sharapov, A. I. Kanaev and A. P. Zakharov // J. Nucl. Mater., 212-215 (1994) 1492-1496.

14. M.R. Louthan, J.A. Donovan, G.R. Caskey// Acta Metallurgica, 23[6] (1957) 745

15. A.V. Kurnaev, N.N. Trifonov // VANT (The Bulletin of a Nucl. Science \& Technique), «Fusion» series. $2002(3-4) 76$.

16. H. Nakamura, T. Hayashi, M. Nishi, M. Arita, K. Okuno // Fus. Engineering \& Design 55 (2001) 513-520.

17. T. Tanabe, Y. Furuyama, S. Imoto // J. Nucl. Mater. 145-147 (1987) 305-308

18. O.V. Ogorodnikova // J. Nucl. Mater. 290-293 (2001) 459-463

19. R. A. Causey, R. A. Kerst, B.E. Mills // J. Nucl. Mater. 123 (1984) 1547-1552. 